\title{
En defensa de la contrastividad en la enseñanza de segundas lenguas
}

Lars Fant ${ }^{*}$

Resumen. Tras décadas de predominio de una ideología que proscribe el uso de la lengua de base en la enseñanza de lenguas extranjeras y reduce la posibilidad de aplicarle un enfoque contrastivo, se reanima hoy día el debate sobre las ventajas y desventajas de una y otra orientación pedagógica. La presente contribución contempla dar una visión de conjunto sobre el campo y abogar por un uso juicioso de la lengua de base así como también por la aplicación de una perspectiva contrastiva, no solo en la clase de lengua extranjera sino también en otros contextos, como el AICLE, la clase de lengua segunda en su comunidad meta, o la enseñanza de lenguas de herencia.

Abstract. After several decades in which the prevailing ideology regarding language use in the foreign language classroom has been to proscribe the use of the $L 1$, a posture which has practically eliminated the possibility of applying a contrastive approach, a new debate has arisen about the pros and cons of the two opposing pedagogies. This contribution aims at presenting an overview of the field as well as giving strong arguments in favor of both a judicious use of the $L 1$ and a contrastive approach in foreign and second language teaching. It is argued that this approach is relevant not only to the FL classroom but also to other contexts such as CLIL education, sL classes and the teaching of heritage languages.

Résumé. Depuis plusieurs décennies de dominance d'une idéologie qui bannit l'usage de la L1 dans l'enseignement des langues étrangères et réduit les possibilités d'application d'une approche contrastive, le débat ressurgit sur les avantages et désavantages des deux pédagogies opposées. L'objectif de la contribution présente est de donner une vue d'ensemble sur ce champ ainsi que de présenter des arguments convaincants non seulement en appui d'un recours judicieux à la L1, mais aussi en défense de l'approche contrastive, dans la classe de langues. II est soutenu qu'une telle approche pourra être appliquée non seulement à la classe LE traditionnelle mais aussi à des contextes tels que l'enseignement EMILE, les classes de langues secondes offertes dans la communauté cible et l'enseignement des langues-patrimoine.

*Departamento de Estudios Románicos y Clásicos de la Universidad de Estocolmo. 


\section{AbreViaturas}

L1 la primera lengua adquirida por el individuo, "lengua materna".

L2 'segunda lengua': cualquier lengua adquirida por el individuo después de la L1.

LE 'lengua extranjera': L2 que se enseña y aprende en contexto escolar fuera de la comunidad meta.

LS 'lengua segunda ': L2 que se enseña y aprende en una comunidad en la que esta lengua es mayoritaria.

LB 'lengua de base': en el contexto de la enseñanza de LE es la lengua de la comunidad mayoritaria, en el de la enseñanza de LS es la L1 (o diferentes L1) de los estudiantes.

LM 'lengua meta': la LE o LS enseñada.

LH 'lengua de herencia': una L1 o L2 temprana adquirida en un entorno en que no es lengua mayoritaria pero sí la lengua materna de los padres o de uno de ellos (la segunda generación “hereda” la lengua de la primera). 
1. INTRODUCCIÓN: ¿HACE FALTA DEFENDER LA CONTRASTIVIDAD EN EL AULA DE LENGUAS EXTRANJERAS?

Lo que hace surgir el asunto de los pros y contras del uso de métodos contrastivos en la enseñanza de lenguas extranjeras (LE) es, por un lado, una sucesiva desvalorización de este enfoque pedagógico a lo largo de las últimas décadas y, por otro lado, un vivo debate que no solo concierne la contrastividad en sí, sino toda la cuestión de la presencia y admisibilidad de la primera lengua (L1) o lengua de base (LB) en el aula de LE.

No tendría sentido intentar contestar a la pregunta de arriba sin aplicar una perspectiva histórica a fin de poder seguir la evolución de las tendencias y modas en la enseñanza de LE. Hace falta ver más de cerca también las tendencias recientes en la evolución de la didáctica de las lenguas extranjeras (LE) y segundas (LS), en particular a la luz del proceso de globalización.

La cuestión de la aplicación o no de métodos contrastivos está íntimamente ligada al debate sobre los pros y contras de recurrir a la alternancia de códigos y uso de la LB en el aula para diversos fines. Aunque es natural asumir que un enfoque contrastivo es promovido por la aceptación del uso de la LB en el aula o tolerancia hacia su uso, es posible imaginar que el método contrastivo podría ser aplicado sin referencia directa a la LB del estudiante y también, por el contrario, que el uso de la LB se admitiría en el aula sin que se aplique ningún enfoque contrastivo.

El contexto en que una y otra cuestión se hace relevante es la clase y aula de LE. Independientemente de que se trate de la enseñanza de español LE, por ejemplo en Suecia, o de inglés LE, por ejemplo en México, el caso prototípico es cuando los alumnos hablan la lengua de la comunidad base y aprenden la de la comunidad meta con un profesor -sea o no hablante nativo de la LB o de la LMquien sabe hacer referencia en el aula a la LB. En cambio, el contexto prototípico para la enseñanza de una LS, por ejemplo la de español para extranjeros en países hispanófonos, es con un alumnado de múltiples L1 que aprende la lengua de la comunidad que los rodea. Aunque estos dos contextos son de naturaleza muy distinta, a la vez de constituir las principales modalidades de enseñanza de lenguas organizadas, raramente han sido objeto de investigaciones científicas comparativas.

\section{TRASFONDO HISTÓRICO}

Hasta los años 70, la enseñanza de LE puso mucho énfasis en la traducción, menos como destreza en sí que como herramienta de aprendizaje. Esa tendencia se veía respaldada por la Hipótesis del Análisis Contrastivo, la 'HAC' (Lado 1957), 
la interpretación "fuerte" de la cual predice que prácticamente todos los errores cometidos en la L2 son atribuibles a la interferencia de la L1. Paradójicamente, la HAC ha servido de respaldo a dos tendencias contradictorias: Por un lado, abre espacio para su aplicación directa en la clase de LE, fomentando una didáctica contrastiva; por el otro lado, da pie para proscribir todo uso de la LB en ella, dado el efecto supuestamente perjudicial de la "interferencia" acarreada por la mezcla de idiomas en el aula.

A partir de los años 70 se empiezan a divulgar diversas teorías sobre el concepto de Interlengua, la más conocida (o al menos más citada) es la de Selinker (1972). Con este concepto se da a entender que lo que se consideraban 'errores' en la producción L2 de hecho forman parte de un sistema coherente propio del aprendiente, sin ser derivables de su L1. En el desarrollo posterior de la teoría de la Interlengua se sustentó que, independientemente de cuál sea su L1, el aprendiente de la L2 sigue una trayectoria más o menos fija y pronosticable que va por etapas. En consecuencia a esta posición se postuló igualmente una 'variedad básica', común a los aprendientes de toda L2 e independiente de su L1 (Klein y Purdue 1992).

Esta visión a su vez fue combinada con las teorías propuestas por Krashen $(1981,1982,1985)$ que distinguen el proceso de 'aprendizaje' del de 'adquisición' además de sustentar la fundamental incompatibilidad de estos dos; al mismo tiempo este teórico supone la existencia de un factor'monitor' del que se vale el aprendiente de una LE o LS para producir lenguaje correcto "controlado" y que permite -aunque solo hasta cierto punto y solo bajo circunstancias favorablesreconvertir algo "aprendido" en elemento "adquirido".

Respaldado por una nueva corriente en el área de la ASL -la interaccionista-, se promovió el enfoque llamado"comunicativo" en la enseñanza de las segundas lenguas (Canale y Swain 1980). Según la visión interaccionista, la exposición a un input comprensible y sucesivamente más manejable, en conformidad con el principio vygotskiano de la Zona de Desarrollo Próximo (ver p. ej. Vygotsky 1978) y combinada con suficiente incentivo a producir output comprensible (Swain 1985), asegurarían una adquisición automática. Anotar que se trata aquí de "adquisición" y ya no de "aprendizaje". Con el enfoque comunicativo se introdujo y se divulgó la idea de que en la medida de lo posible hay que evitar el uso de la LB en el aula LE.

En los últimos años hemos visto surgir tendencias nuevas y a veces contradictorias. Por un lado, parece borrarse en grado creciente el límite entre la enseñanza de LE y la de LS, al propagarse la idea de que en un mundo cada día más globalizado, el alumnado de las LE sea tan heterogéneo en cuanto a sus diversas L1/LB que ya se puede proceder como si el aula fuera de LS (una tendencia que se nota en particular en muchos países europeos marcados por 
recientes olas migratorias y el influjo de refugiados provenientes sobre todo de Medio Oriente). Esta visión lógicamente va acompañada de una desestima del respaldo que la referencia a la L1 del estudiante pudiera constituir para su aprendizaje de la LM.

Por otro lado, varios estudios subrayan la utilidad de recurrir a la alternancia de códigos, tanto en el aula multicultural como en las clases de AICLE (Aprendizaje Integrado de Contenidos y Lenguas Extranjeras) o clases de inmersión -y, por extensión, también en el aula de LE convencional. Respecto de las clases de inmersión y de AICLE se ha producido bastante polémica: la postura predominante entre los profesionales del área (o sea, tanto los profesores como los ideólogos de la didáctica) es que el uso de la LB contrarresta los principios básicos de la inmersión (Turnbull y Dailey-O'Cain 2009:3). Opuestos a estas voces se alzan otras agregando cada vez más argumentos en apoyo al uso de la LB en contexto AICLE. Sin embrago, antes de proceder al examen de la enseñanza de este tipo, detengámonos de momento en la situación tal como se presenta en la clase de LE tradicional.

\section{Posiciones tOMAdAs CON RESPECTO AL USO DE LA LB EN EL AULA DE LE}

\section{A. Posturas de rechazo}

Han prevalecido en el debate una postura negativa frente al uso de la LB en el aula de $L E$, al ser recomendado o prescrito el uso exclusivo de la LM para todas las funciones educativas. Los principales argumentos presentados parecen ser los siguientes (cf. Macaro 2001):

- Hay pocas oportunidades de input fuera del aula.

- La LB es fuente de interferencias perjudiciales a la adquisición.

- El camino evolutivo de la adquisición de todas maneras no se deja influir por informaciones o instrucciones dadas por el profesor (postura calificable de "derrotista", basada en interpretaciones fuertes de la teoría de Krashen 1981, etc. relativa a la oposición adquisición-aprendizaje).

- Solo la exposición a input comprensible junto con el incentivo a producir output comprensible pueden garantizar una adquisición eficiente. 
Al respecto, un teórico dedicado a cuestiones de adquisición bilingüe, Fred Genesee, aboga por el uso exclusivo de la LM como principio de base por ser "el enfoque más eficiente en la enseñanza de las segundas lenguas, en el contexto escolar" (Genesee 1994: 6). Por otro lado, Krisztina Nagy y Daniel Robertson, dos estudiosos que expresan dudas hacia el principio de la exclusividad de la LM, constatan que esta tendencia prevalece actualmente en la enseñanza de lenguas:

As part of the communicative approach (Breen \& Candlin, 1980; Canale \& Swain, 1980; Littlewood, 1981) to foreign language teaching, the teacher is usually expected to use the target language as much as possible to provide comprehensible input for the learners. (Nagy y Robertson 2009: 66).

\section{B. Sin tomar posición}

Varios estudiosos y profesionales se abstienen de posicionarse al respecto. De hecho, los teóricos de las diferentes escuelas del campo de la ASL casi nunca abogan explícitamente por la proscripción de la LB en el aula de la LM o mejor: la cuestión ni siquiera es abordada o mencionada. Así va entre los generativistas (p. ej. Chomsky 1965), quienes estiman que el aprendizaje de segundas lenguas se deriva de funciones cerebrales innatas ("parameter setting"). También se da la actitud de no tomar posición en el campo funcionalista, en el que se estima que la L2 se adquiere a través de input premodificado (Krashen 1985), así como en el interaccionista, en el que se sustenta que la $L 2$ se adquiere a través del input interaccionalmente modificado (Ellis et al. 1994; Long 1981) o por el output forzado (Ellis \& He 1999; Swain 1985).

Es notable que en estudios dedicados a los tipos de retroalimentación dada respecto a los errores cometidos por los aprendientes, se afirma que la segunda lengua se adquiere a través de evidencia negativa al igual que positiva (DeKeyser 1993), no se hace ninguna mención sobre la lengua en la cual se suministra uno y otro tipo de feedback.

Se puede concluir que para estos autores, el uso de la LB se considera simplemente innecesario, o se hace caso omiso de la cuestión, razón por la cual no es de extrañar que los profesionales vean poco interés en fomentar el uso de la LB en el aula (Macaro 2009: 36-37).

Rod Ellis, influyente teórico de la adquisición de segundas lenguas, tampoco toma posición en lo que se refiere al uso a la LB en el aula LE. Afirma que la aplicación de esta práctica representa un asunto controvertido y complejo, "dado que el grado de utilidad del uso de la LB depende del contexto didáctico" (Ellis 2008: 801).

Otro ejemplo significativo de la eliminación del asunto del orden del día lo ofrece el programa paneuropeo VILLA sobre el aprendizaje incipiente de LE, proyecto 
de gran escala realizado bajo condiciones experimentales controlados (Dimroth et. al. 2013). En este proyecto, que consiste en enseñar polaco a principiantes de diferentes países europeos con diversas $L B$, no se hace ninguna mención del uso de la LB en el aula. Probablemente la interpretación fuerte del principio de enseñanza comunicativa -o sea, la abstención total de recurrir a la LB- se ha dado simplemente por descontada por los organizadores de ese proyecto.

\section{La posible convertibilidad del "aprendizaje" en "adquisición"}

Una forma extrema de interpretar a Krashen $(1981,1982,1985)$ es sostener la total incompatibilidad entre 'aprendizaje' y 'adquisición'. Esta idea se ve respaldada por la teoría de Paradis $(2004,2009)$ acerca de la distinción 'conocimiento declarativo'/ 'facultad procedimental'. Este último autor argumenta (con apoyo ciertamente contundente en estudios neurofisiológicos y neurolingüísticos) que lo que denomina'conocimiento declarativo'y'facultad procedimental'se situán en centros cerebrales distintos y corresponden a mecanismos separados en la producción de lenguaje: las producciones gobernadas por la facultad procedimental son automáticas y no sometidas a introspección mientras que las gobernadas por el conocimiento declarativo son monitoreadas de manera consciente.

Aplicada la teoría de Paradis $(2004,2009)$ sobre la situación del aprendiente de una $L M$, es natural deducir que el usuario no demasiado avanzado del nuevo idioma tiene que hacer frecuentes consultas a su conocimiento declarativo. En cambio, un hablante nativo lo hace solo en contados casos (ante todo cuando tiene que premeditar su discurso y en particular a la hora de escribir), dado que su grado de automatización (o sea, acceso directo a la facultad procedimental) en la producción oral, así como muchas veces en la escrita, es muy elevado. Debemos reconocer, por otra parte, que los usuarios avanzados de LM, aunque sin casi nunca alcanzan niveles nativos, también alcanzan un alto grado de automatización productiva.

El paralelismo con Krashen consistiría en ver el 'aprendizaje' como ligado enteramente a la función 'conocimiento declarativo' y 'adquisición' como vinculado a la'facultad procedimental.' Ahora bien, si los elementos "aprendidos" nunca llegaran a ser elementos "adquiridos", dada la supuesta incompatibilidad de uno y otro mecanismo, enseñar una segunda lengua de manera deliberada no tendría sentido si no fuera por la pura exposición del aprendiente a input, sin intento por parte del docente de dar explicaciones.

Esta idea va en contra, no solo de la intuición de la gran mayoría de docentes de segundas lenguas, sino también de una serie considerable de estudios científicos. Una visión alternativa -así como interpretación más realista de Paradis (2004, 2009)- consistiría en atribuir al conocimiento declarativo la 
capacidad de incidir bajo ciertas condiciones en la facultad procedimental. El mecanismo es bastante sencillo: el individuo que es concientizado sobre la existencia de una cierta estructura (palabra, locución, construcción etc.) adquiere al mismo tiempo una herramienta para entrenarse a sí mismo a fin de terminar por automatizar la estructura en cuestión.

Así se justifican también los actos de aloretroalimentación y alocorrección: dado que no se puede influir directamente en la facultad procedimental del aprendiente sino solamente en su conocimiento declarativo, tiene sentido que el profesor de la LM (o usuario más avanzado de la misma) alocorrija al aprendiente (usuario más débil) y la forma supuestamente más eficaz de hacerlo es a través de referencias a la LB del aprendiente (usuario más débil) de la LM.

Es un desafío pedagógico-didáctico adecuar la manera de dar expresión a tales actos de alocorrección al nivel evolutivo (en el proceso adquisicional de la LM), intelectual, social y cultural del aprendiente. Es también un desafío pedagógico-didáctico revisar, siempre y cuando resulte necesario, las rutinas didácticas establecidas.

Paradójicamente, desde esta perspectiva, el modelo de Krashen, el cual originalmente sirvió para apoyar el uso exclusivo de la LM en la clase de LE, puede usarse ahora para dar respaldo a que se recurra a la LB (Norrman 2014).

\section{Posturas de aceptación}

A pesar de las numerosas posturas negativas o indiferentes, también se pueden encontrar argumentos que prestan apoyo directo o indirecto al uso de la LB en el aula de LE. Una perspectiva interesante la abre la teoría del Procesamiento Cognitivo (ver p.ej. Nick Ellis 2005), según la cual la L1 y la (o las) L2 no están almacenadas por separado. Según Nick Ellis (2005), el léxico mental se deja mejor representar como un conjunto de conexiones (cada vez creciente a lo largo de la vida del individuo) y potenciales activaciones de las mismas, que no pertenecen a ninguna lengua específica hasta el momento de la formulación. Dado que las conexiones establecidas con la L1 son mucho más fuertes que las establecidas con la o las L2, el hacer caso omiso de la primera en el proceso de aprender la segunda es desechar una herramienta esencial que está a la disposición del aprendiente.

Por otra parte, dentro del marco de la Teoría Sociocultural (Vygotsky 1978) se sustenta que la voz interna y el habla privada conforman la base de nuestro pensamiento y que casi siempre se realizan en la lengua nativa (ver p.ej. Brooks y Donato 1994).

Diversos estudios sobre el bilingüismo nos brindan otra perspectiva más sobre la cuestión. Así, un trabajo reciente (Bylund et. al. 2012) que analiza la 
actuación de individuos bilingües residentes en Suecia (con el español como L1 y el sueco como L2), muestra claramente que el sostenimiento de la L1 va de la mano con un mejor desarrollo y dominio de la $L 2$.

A estas alturas cabe preguntarse de qué manera, o maneras, contribuye el uso de la LB a mejorar el aprendizaje. Un concepto clave al respecto es la alternancia de códigos. Así, Dailey-O'Cain y Liebscher (2009: 143) afirman que "el admitir la alternancia de códigos por parte de los estudiantes así como del profesor puede respaldar el aprendizaje a través del andamiaje o al promover la intersubjetividad". Macaro (2009:38), por su parte, sustenta que el uso óptimo de la alternancia de códigos es el que se realiza en lo que llama "el aula comunicativa en sentido amplio" (the broadly communicative classroom). Tal práctica fomentaría la adquisición en mayor medida que el uso exclusivo de la LM.

Este último autor advierte además contra el riesgo de que el profesor renuncie a transmitir información transcendente simplemente por considerarla demasiado difícil para los aprendientes si es expresada en la LM, lo cual no sería más que una estrategia elusiva ("avoidance strategy"; Macaro 2009: 40) por parte del docente. A través de dos estudios cuantitativos y cuasi-experimentales, el mismo autor (Macaro 2009) presenta resultados que corroboran la idea de que el uso de la LB en el aula fomenta el aprendizaje de vocabulario de la LM:

...we have some evidence that some items of vocabulary might be better learnt through a teacher providing first-language equivalents because this triggers deeper semantic processing than might occur by providing secondlanguage definitions or paraphrases. (Macaro 2009: 48).

Otras voces se alzan en contra del uso exclusivo de la LM en el aula de LE. Tal exclusividad, según Macaro (2001:545) puede generar una exagerada simplificación del lenguaje así como una muy alta dependencia de los cognados. El mismo autor advierte contra el riesgo de que, en la enseñanza impartida exclusivamente en la LM, los ejercicios de comprensión oral tengan como único objetivo la comprensión de lo que dicen los hablantes, y no la adquisición del vocabulario o estructuras gramaticales representados en el texto (Macaro 2009: 48).

Dos otros estudiosos de la cuestión dan la siguiente recomendación explícita:

Talking less about specific classroom practices and more about classroom philosophy: There is simply no evidence that a prescribed target-language only environment is beneficial to learners, and there is ample evidence that it may be detrimental. (Turnbull y Dailey-O'Cain 2009).

Podría enumerarse una serie de funciones importantes que cumple el uso de la LB en la clase de LE: 
- Se usa para fines organizativos (resulta más práctico organizar la actividad en la LB).

- Constituye un beneficioso atajo para dar explicaciones, al usarse la LB como metalenguaje, así como para retroalimentar o corregir.

- La cuarta destreza según el MCER (Council of Europe 2001) -la mediación- requiere un uso consistentemente simultáneo de la LB y LM.

- Conectar cognitivamente la LM con la LB es una manera de derribar los "compartimientos estancos" entre una y otra: en eso residiría la auténtica contrastividad (cf. Gajo 2015).

Un aspecto importante, además de los factores arriba enumerados, es que el uso de la LB en la enseñanza de las LE (al igual que, como argumentaremos más adelante, en la de LS) fomenta la formación de una identidad bilingüe. Hay que reconocer el hecho de que los bilingües no se comportan como los monolingües. Para un niño bilingüe (tratándose tanto de bilingüismo simultáneo como sucesivo) es natural valerse de la alternancia de códigos para estructurar la conversación así como para construir una identidad social (Fuller 2009: 129).

En consecuencia, al aprendiente de una LE o LS le debe ser permitido practicar una conducta de bilingüe al usar sus dos o varios idiomas "en su pleno repertorio, esto es, practicando la alternancia de códigos" (Fuller 2009: 115-6). Asimismo, cabe considerar los aprendientes de una LE o LS lengua, no como hablantes imperfectos de la LM, sino como incipientes bilingües (Turnbull y Dailey-O'Cain 2009:186), guardando en mente que los aprendientes nunca llegarán a ser hablantes monolingües perfectos: "Los profesionales que se fijan ese objetivo están condenados al fracaso" (Turnbull y Dailey-O'Cain 2009:186).

\section{E. Comparación de clases en las que se recurre o no a la LB}

Es notable que se hayan producido muy pocos estudios dedicados a la comparación de la enseñanza de LM realizada con o sin recurso a la LB. Posiblemente se haya considerado irrelevante o incluso inoportuna la cuestión, ante todo por ser visto el enfoque contrastivo como "pasado de moda".

Admitamos que los estudios de este tipo son difícilmente realizables en las aulas de LE por encontrarse, en la realidad, pocos ejemplos de (la prescrita) exclusividad de la LM que hayan sido llevados a la práctica, por muy 
recomendada e incluso prescrita que ha sido. Tampoco son fáciles de realizar en las aulas de LS, dado que los grupos suelen ser mixtos, con una variedad de LB que el profesor no siempre (o casi nunca) conoce.

Destaca un trabajo sumamente interesante que indirectamente permite sacar conclusiones respecto de la aplicación de uno u otro enfoque: un estudio (Bardovi-Harlig y Dörnyei 1998) en el que se compararon los progresos hechos por alumnos de inglés LE en Hungría con los de alumnos de inglés LS en Estados Unidos, de diversas L1. La enseñanza de LE realizada en Hungría era de tipo tradicional, o sea, con frecuente uso de la LB, mientras en las clases de LS estudiadas en Estados Unidos no se hacían referencias a ninguna LB. El resultado principal del estudio es que los aprendientes de inglés LE mejoraron sus conocimientos gramaticales en mayor medida que los aprendientes de inglés LS, mientras lo contrario fue el caso con respecto a las destrezas pragmáticas.

Una conclusión del estudio de Bardovi-Harlig y Dörnyei (1998) es que para mejorar las destrezas pragmáticas sirve la interacción, de preferencia realizada en la comunidad meta, mientras que para mejorar los conocimientos estructurales sirve un enfoque contrastivo.

\section{TRES CONTEXTOS PARTICULARES}

Hasta este punto, nos hemos dedicado a dilucidar argumentos en pro y en contra del uso de la LB en la clase de LE, sin distinguir el uso contrastivo de otros usos de la LB. En los apartados que siguen examinaremos la enseñanza de LM en tres contextos que difieren de la clase LE tradicional. Comenzaremos por abordar (1) la enseñanza de LE o LS en el aula multilingüe y multicultural, seguiremos por analizar (2) aspectos del uso de la LB en la clase de inmersión o de AICLE, y terminaremos por examinar la aplicación de un método contrastivo en la enseñanza de lenguas de herencia (LH).

\section{A. La enseñanza de LE o LS en el aula multilingüe y multicultural}

No existen muchos estudios que tematizan el uso de la LB y el enfoque contrastivo en el aula multilingüe y multicultural. Dos trabajos de fecha reciente realizados en Suecia merecen ser comentados. Uno, que versa sobre la alternancia de códigos practicada en una clase de inglés $L E$, en la cual los 40 participantes eran niños de 15 diferentes lenguas nativas distintas al sueco (Lugoloobi-Nalunga 2013), muestra resultados interesantes. El profesor, quien no conocía estas lenguas pero sí el sueco, permitía que los alumnos se comunicaran entre ellos en sus respectivas $L B$, interviniendo a veces en sueco, a veces en inglés y creando 
así un clima en el que era natural ver cómo las diversas lenguas diferían entre sí. La autora constata, por un lado, que la alternancia de códigos es un fenómeno espontáneo y natural en el desarrollo de la LM. Sus resultados muestran además que cuando la alternancia de códigos se combina con un enfoque contrastivo, el efecto es positivo para el aprendizaje de la LM en varios planos. En particular fomenta el desarrollo de vocabulario y el concomitante desarrollo conceptual, y también parece satisfacer las necesidades de aclaración y puesta de relieve que experimentan los alumnos.

El segundo estudio, dedicado a la enseñanza de pronunciación en clases de sueco LS (Tronnier y Zetterholm 2015), muestra cómo los aprendientes, que representaban cuatro L1 tipológicamente distintas del sueco, pudieron mejorar significativamente su manejo del acento de palabra en la LM después de haber sido grabados y tras haber podido escucharse a sí mismos con andamiaje por parte del profesor. Éste, sin saber ninguna de las LB de los alumnos, había sido instruido previamente sobre los patrones de acentuación de las respectivas lenguas.

La conclusión de ambos estudios es que la alternancia de códigos y el enfoque contrastivo pueden ser practicados con éxito aun en clases multiculturales y multilingües en las que el profesor no sabe las LB de sus alumnos.

\section{B. El uso de la LB en la clase de inmersión o de AICLE}

Al igual que en el caso de la clase de LE tradicional predomina la postura crítica hacia el uso de la LB -por lo menos al nivel oficial. Cabe citar lo que dice al respecto la fundación canadiense para la educación de las Provincias del Atlántico':

L'apprentissage doit être intensif sans toutefois être une noyade. Les élèves doivent très tôt pouvoir comprendre le français et l'utiliser pour communiquer. II est donc essentiel que la seule langue de communication dans la salle de classe soit le français. ${ }^{2}$

(La Fondation d'éducation des provinces de l'Atlantique, 1997: 9).

1 Estas provincias son Quebec y Nueva Brunswick en las que el francés es lengua mayoritaria (Quebec) o fuertemente representada (N. Brunswick); la cita se refiere a las clases de inmersión destinadas principalmente a estudiantes angloparlantes.

2 "El aprendizaje debe ser intensivo sin por eso llegar a ser un hundimiento. Los alumnos desde una fase temprana deben poder entender el francés y utilizarlo para fines comunicativos. Es por lo tanto esencial que el único idioma utilizado para la comunicación en el aula sea el francés." 
Entre los profesores canadienses parecen coexistir tres posturas distintas frente al uso de la LB en la clase de inmersión (Macaro 2009; McMillan y Turnbull 2009). La voz más "ortodoxa" declara que la LM solo puede ser adquirida a través de la LM; el uso exclusivo de la LM daría lugar a una "realidad virtual" que reflejara la sociedad meta desde la perspectiva de sus hablantes nativos así como de los inmigrantes. La segunda postura, que supone una adaptación del principio a la realidad, consiste en decir que la LM, en principio, se aprende a través del uso único de la LM pero este es un ideal difícil de alcanzar, por lo cual hay que cometer "el pecado" de recurrir de vez en cuando a la LB. Según la tercera postura -más resignada, o más adecuada a la realidad, si preferimos- se supone que el uso de la LB, practicado con moderación, puede facilitar y fomentar el aprendizaje de la LM:

Some [teachers] believed that there was some recognizable value in first language use. That at certain moments during the teaching and learning process the use of the first language might actually enhance learning more than by sticking to the second language. I labelled this position the 'optimal position'. (Macaro 2009:36).

No brillan por su ausencia los argumentos aducidos a favor del uso de la LB en la clase de inmersión. Jim Cummins, en su estudio sobre la educación bilingüe (2010), declara que la práctica del uso exclusivo de la LM en el aula genera una pedagogía cualitativamente inferior que favorece el entendimiento literal a expensas de la comprensión crítica:

...the principle of language separation and vestiges of 'direct method' teaching approaches (i.e. remaining totally in the target language) in immersion programs thus sometimes results in pedagogy that is less cognitively challenging and creative than many educators would consider appropriate. The provision of comprehensible input in the second language is interpreted as the promotion of literal rather than critical comprehension. (Cummins 2000: 10).

Asimismo, tres investigadores canadienses (Behan, Turnbull y Spek 1997) que realizaron un estudio con base en grabaciones de estudiantes de inmersión francesa del séptimo grado, concluyeron que:

L1 use can both support and enhance L2 development, [both languages] functioning simultaneously as an effective tool for dealing with cognitively demanding content. (Behan et al 1997: 41)

En la misma línea, Swain y Lapkin (2000), en un estudio sobre la actuación de estudiantes de inmersión francesa del octavo grado, encontraron que los participantes tuvieron mejores resultados en tareas colaborativas cuando se les permitía usar en cierta medida su LB. Estos estudiantes usaron la LB en 
aproximadamente $25 \%$ de los turnos, de los cuales una parte mínima tenía referencia externa a la tarea y los más sirvieron para importantes funciones sociales y cognitivas. Los autores concluyen que el uso juicioso de la LB apoya claramente el aprendizaje y uso de la LM (Swain y Lapkin 2000:268).

En este contexto cabe mencionar el concepto de translanguaging, acuñado por García (2008) y que refiere al uso estratégico de la alternancia de idiomas para realizar actividades diferentes. Esta práctica aplicada a la enseñanza de LM podría usarse alternando la LB con la LM por ejemplo según la modalidad usada (o sea, escritural/oral; García 2008: 45). Más importante aún, la autora afirma que este concepto de translanguaging pone en evidencia lo ya sugerido por cognitivistas como Nick Ellis (2005) de que no existen límites exactos entre las lenguas de los bilingües (García 2008:47).

La secuencia que sigue, grabada en el contexto de enseñanza de inmersión francesa de nivel universitario, en una clase de derecho en la que los estudiantes eran germanoparlantes, ilustra bien el funcionamiento del translanguaging así como la aplicación de un enfoque lingüístico contrastivo sin que el aula sea de LM ( $\mathrm{P}=$ profesor; $\mathrm{E}=$ =studiante; los tramos en alemán aparecen en cursiva y la traducción del diálogo entre corchetes $)^{3}$ :

P: Quel est grand principe inscrit même dans la convention des droits de I'homme sur la manière dont quelqu'un est traité quand on lui reproche un crime ou un délit ? [¿Cuál es el gran principio inscrito incluso en la convención de los derechos humanos acerca de cómo se trata a alguien acusado de un crimen o delito?]

E: Unschuldsvermutung [Presunción de inocencia]

P: Comment est-ce qu'on appelle ça en français ? [¿Cómo se llama eso en francés?]

E: La preuve Unschuld ? [¿La prueba Unschuld (=inocencia)?]

P: $\quad$ Non. La preuve ce serait justement ... [No. La prueba sería justamente...]

E: Der Beweis. [La prueba.]

P: Oui mais la Vermutung [suposición], c'est quoi ? [Sí pero la Vermutung qué es?]

E: Soupçon. [Sospecha.]

P: $\quad$ Le soupçon c'est aussi une Vermutung [suposición] mais une Vermutung, eine negative Vermutung [una suposición negativa]. [La sospecha es también una Vermutung pero una negative Vermutung.]

3 La secuencia proviene de los materiales del lingüista suizo Laurent Gajo (Gajo 2015), quien amablemente me ha autorizado a usarlo en el presente artículo. 
P: $\quad$ Und das nennt man wie wenn man vermutet dass etwas schief ist [Y ¿eso se Ilama cómo cuando se supone que algo va mal], wie ist das auf deutsch? [¿cómo se dice eso en alemán?]

E: Ein Verdacht. [Una sospecha.]

P: Aber die Unschuldsvermutung, man könnte nicht gut von einem Unschuldsverdacht sprechen, nicht, [Pero, la Unschuldsvermutung, no se puede realmente hablar de una "sospecha de inocencia" ¿no?]

Und die Unschuldsvermutung auch auf französisch braucht man eben ein anderes Wort für Vermutung, nicht 'soupçon' sondern... [Y la Unschuldsvermutung también en francés hace falta usar otra palabra para Vermutung, no'soupçon/sospecha' sino...]

E: C'est la présomption d'innocence. [Es la presunción de inocencia.]

A lo largo de esta secuencia se ve cómo el profesor lleva al estudiante a tomar conciencia, paso por paso, no solo del significado de ambos componentes del compuesto nominal alemán Unschulds-vermutung, sino también del de ciertas nociones afines ('prueba' frente a 'suposición'y 'suposición' frente a 'sospecha') así como del de cada una de las palabras francesas que conforman la locución que corresponde a'presunción de inocencia'. Huelga decir que un efecto comparable no podría haber sido alcanzado al valerse únicamente de la LM francesa.

\section{El enfoque contrastivo en la enseñanza de las lenguas de herencia (LH)}

Un caso particularmente delicado lo representa la enseñanza de $\mathrm{LH}$, para la cual haría falta hacer referencia tanto a la lengua mayoritaria (en el nivel en que lo domine el aprendiente) como a la $\mathrm{LH}$ al poner particular énfasis en las particularidades de esta en comparación con la LM estándar. O sea: hace falta aplicar a este tipo de clase una visión doblemente contrastiva.

La siguiente definición del usuario de LH, acuñada por Valdés (2002), es la más comúnmente aplicada en Estados Unidos, nación en la cual el español por motivos históricos es -y con mucho- la lengua minoritaria, y con eso la LH, más divulgada. Según esta definición, un hablante de una LH es una persona "criada en un hogar en el que se habla otro idioma que el inglés, quien habla este idioma -o al menos lo entiende- y que en mayor o menor medida es usuario bilingüe de este idioma y el inglés" (Valdés 2002:2).

Para el profesor de LE, el estudiante de LH difiere en importantes respectos del aprendiente tradicional de LE. De hecho, los aprendientes de una LH se encuentran en una posición diametralmente opuesta a la de los aprendientes de una LE: mientras estos se valen de la gramática para acceder a la lengua, 
aquellos recurren a su lengua e intuiciones respecto de ella para acceder a su gramática mental (Parodi 2001).

Cabe destacar que los usuarios de una lengua de herencia se sitúan dentro de una escala continua de proficiencia: el usuario muy poco proficiente puede haber tenido una exposición muy limitada a la lengua en su niñez, y a lo mejor no ha sabido hablarla nunca. Al otro extremo encontramos a usuarios que pueden pasar por hablantes nativos monolingües. Se considera que la desigualdad que caracteriza a este grupo, visto como población estudiantil, así como la disparidad de las competencias que llevan al aula, hacen que los tests tradicionales usados para medir el nivel de ingreso de aprendientes LE resulten poco apropiados (Fairclough 2012:268).

Hay que guardar en mente también que el usuario típico de español LH suele hablar un dialecto regional y que normalmente no ha sido expuesto a otras variedades sino de manera muy esporádica. Los docentes de estas clases deberán, por lo tanto, ayudar al estudiante a comprender la importancia de acceder a una variedad más estandarizada del español, a la vez de respetar su variedad particular en cuanto marca de identidad grupal -lo cual representa un equilibrio delicado para mantener. La siguiente lista (fuentes: Valdes 2012; Villa 2002) enumera los objetivos deseables más destacados para la enseñanza de español LH, en Estados Unidos y, por extensión, en el mundo:

- La adquisición de una variedad del español más prestigiosa;

- Una expansión del abanico bilingüe, o sea, del continuo de habilidades y estrategias que pueda poseer el individuo en cada lengua;

- La transferencia al español de una literacidad ya adquirida en inglés;

- El fomento de una actitud positiva frente a la lengua y cultura de herencia;

- El desarrollo de una conciencia tanto intra como intercultural;

- El desarrollo de una identidad colectiva y personal en el estudiante que se conecte con su cultura de origen.

\section{El ORIGEN DE LOS “ERRORES” LINGÜÍSTICOS Y PRAGMÁTICOS}

Recordemos que la preferencia por la exclusión de la LB en el aula LE radica fundamentalmente en la idea de la interlengua como sistema en alto grado 
autónomo, combinándose la autonomía adjudicada a este sistema interino con la infravaloración del factor transferencia desde la L1.

A pesar del indiscutible peso que tiene la interlengua en la producción de errores o anomalías en la LM, hay que reconocer que la transferencia -ya sea positiva o negativa- resulta ser un factor muy potente por un motivo contundente: cuando una determinada categoría (principio, pauta) de la LM no ha sido internalizada por el aprendiente y el contexto le obliga a "improvisar", ¿de dónde va a sacar las herramientas necesarias? La respuesta es obvia: los recursos no pueden provenir sino de los conocimientos ya adquiridos por el individuo, o sea, de su L1 o, cuando el caso se da, de otra u otras L2 anteriormente aprendidas.

El mecanismo de la transferencia opera en todos los planos lingüísticos y comunicativos afectando todos los tipos de habilidad: desde la fonética segmental y suprasegmental hasta el nivel sociocultural, pasando por el léxico, las unidades fraseológicas y la gramática. Recordemos, sin embargo, que la interlengua incide en mayor grado en la gramática (morfología y sintaxis) que en otros componentes. En contraste, en fonética y fonología el factor transferencia parece desempeñar el papel predominante (Major 2001).

El reconocimiento de la importancia central del factor transferencia nos lleva a tomar conciencia de otro hecho, que a algunos les podría parecer obvio pero a todos, a saber: al estudiante que no tome conciencia de las transferencias que ha internalizado y produce, le va a faltar una importante herramienta para corregirse y apropiarse de un uso más adecuado e idiomático.

\section{DOMINIOS PARA RETROALIMENTACIÓN CONTRASTIVA}

El objetivo de este penúltimo apartado es ilustrar cómo el factor transferencia genera anomalías en un amplio espectro de planos lingüísticos. Decimos "anomalías", dado que en la mayoría de los ejemplos citados, las transferencias desde la L1 no producen enunciados agramaticales sino estructuras poco idiomáticas en la $L M$, sin respetar las convenciones de la $L M$, o simplemente secuencias desacertadas, al expresar un contenido más o menos distinto del mensaje contemplado. Los ejemplos han sido elegidos del uso nativo y no nativo del español, inglés y sueco.

\section{A. Fonética suprasegmental}

El ejemplo que sigue tiene que ver con la interpretación de los tonos de frontera que aparecen con frecuencia en el español L2 producido por suecoparlantes. Se 
produce al final del grupo tonal una subida de tono, transferida del sueco L1, la cual corresponde a una función de cortesía destinada a atenuar la imposición y reforzar la autoimagen positiva (Aronsson y Fant 2014):

Quisiera reservar una mesa para cinco personas para las ocho $\uparrow$.

Lo que un hispanohablante inadvertido entenderá es una señal que expresa, bien sea una solicitud de información ("tono interrogativo"), o bien que el hablante desea proseguir con su turno ("tono continuativo"). Concientizar al aprendiente que usa esta subida de tono mal adecuada al uso nativo es suministrarle una herramienta para autocorregirse y para evitar malentendidos pragmáticos, que, a pesar de ser normalmente subliminales, no pasan desapercibidos y son capaces de reducir la eficiencia de la comunicación.

\section{B. Léxico}

Un área en la que resulta natural practicar un enfoque contrastivo para concientizar al aprendiente de sus errores o anomalías son los llamados falsos amigos. La concientización funciona de manera bidireccional, como se ve en los ejemplos siguientes relativos a la pareja español-inglés. Así, decir en inglés:

how will you dispose of the fortune that your grandfather left you?

no traduce el sentido ¿¿cómo vas a disponer de la fortuna que te legó el abuelo?' sino que transmite al oyente nativo la idea de que habría que quitarse la fortuna de encima. Inversamente, decir en español:

después de terminar de escribir el artículo voy a disponer de todas las notas.

(si es que se entiende; el enunciado a un hispanohablante le parecerá un tanto raro) no traduce 'after I've finished writing the paper I will dispose of all the notes'. En vez de 'disponer de' debería haberse usado el verbo 'deshacerse de' o 'botar'.

\section{Unidades fraseológicas}

Las colocaciones de la LM suelen constituir un área de difícil aprendizaje, donde abundan las instancias de transferencia desde la L1. El ejemplo que sigue concierne al verbo que debe ir con el sustantivo 'decisión' y equivalentes vocablos en sueco ('bes/ut') e inglés ('decision'). En este caso, el español y el sueco coinciden en usar el verbo 'tomar' y sus equivalentes en sueco 'fatta' o 'ta'. En 
inglés, por el contrario, se recurre al verbo 'make', o sea 'hacer'. En consecuencia, el factor transferencia puede generar estructuras anómalas como:

*hicimos una decisión importante (enunciado que podría ser producido por un angloparlante en su español L2)

*we took an important decisión (enunciado que podría ser producido por un hispano- o suecoparlante en su inglés L2)

*vi gjorde ett viktigt beslut ('hicimos...'; enunciado que podría ser producido por un angloparlante en su sueco L2.)

Difícil será corregir errores de este tipo sin hacer una comparación con el uso de la $L 1$ respectiva, o al menos mucho más complicado si se aplica estrictamente el principio de la exclusión de la LB en el aula.

\section{Morfología}

Un fenómeno gramatical del español capaz de producir estructuras anómalas cuando es transferido a otra lengua es aquel uso modal del futuro flexionado que suele ser denominado "futuro de probabilidad". Esta extensión del campo semántico de un morfema que expresa referencia temporal para cobrar el sentido epistémico de 'probablemente $X$ ', 'seguramente $X^{\prime}$, o 'se puede inferir que $X^{\prime}$ no tiene correspondencia en, por ejemplo, el inglés. Así, un usuario nativo del español podría formular en su inglés $L 2$ algo como:

*they will have taken that into consideration

para expresar un mensaje tranquilizador equivalente a'ya lo habrán tomado en consideración'. El mismo hablante hispanófono de inglés L2 sería también capaz de decir:

\section{*you will be right}

para formular un enunciado de interpretación concesiva que en español se plasmaría como 'tendrás razón'. Ambos enunciados, en su contexto, producirá una impresión de rareza en un oyente usuario nativo del inglés. Un efecto más 
sutil produciría un angloparlante que en su español $L 2$, en vez de usar un futuro de probabilidad, recurriría a adverbios como 'probablemente' o 'seguramente':

seguramente lo han tomado en consideración probablemente tienes razón.

Estos dos enunciados, aunque perfectamente gramaticales y admisibles, no evidencian el mismo nivel de idiomaticidad que 'lo habrán tomado en consideración' o 'tendrás razón' y junto con otros indicios podrían contribuir a una impresión de "acento extranjero". Otra vez, explicar esto haciendo referencia a la L1 del emisor simplifica mucho las cosas en comparación con la compleja explicación que haría falta dar si solo se permitiera referirse a la LM.

\section{E. Sintaxis}

Aunque la gramática esté menos expuesta a las transferencias negativas que por ejemplo la fonética, cabe hacer hincapié en el hecho de que diferentes lenguas tienen preferencias distintas a la hora de crear estructuras sintácticas. Un ejemplo de esto es la selección de sujeto gramatical entre diferentes referentes candidatos. El inglés y el sueco tienen una más marcada preferencia que el español por elegir un referente humano no impersonal para ocupar el cupo del sujeto, razón por la cual se dice, en inglés y en sueco:

I got the job (inglés)

jag fick jobbet (sueco),

o sea, literalmente 'recibí el contrato', para expresar un contenido que un hispanohablante nativo probablemente formularía como 'me dieron el contrato'. Otra vez vemos que la transferencia de la pauta $L 1$ a la $L 2$ produce, ya no estructuras agramaticales pero si anómalas en el sentido de que les faltan idiomaticidad. 'They gave me the job' simplemente no suena en inglés igual de bien que 'I got the job'. Una explicación de enfoque contrastivo le facilitaría sin duda al aprendiente la tarea de apropiarse esta distinción.

\section{F. Pragmalingüística}

La expresión lingüística mediante la cual se transmite un determinado contenido pragmático raramente es totalmente análoga en dos idiomas distintos. A modo de ejemplo podemos citar el giro inglés 'why don't you (we, etc)' que es una opción no marcada para iniciar una sugerencia. Existe también en el español un 
giro parecido, '¿por qué no...', el cual no da expresión a un contenido idéntico al de la construcción inglesa, sino que expresa un mayor grado de insistencia al indicar que la sugerencia que sigue debería serle obvia al destinatario. Si un angloparlante en su español L2 dice '¿por qué no te sientas un rato a descansar?' para transmitir un contenido que en su lengua nativa habría formulado 'why don't you sit down and rest for a while', ciertamente será entendido por un interlocutor usuario de español L1, aunque a éste le pueda parecer un poquitín exagerada la manera de expresarse. Debemos tener en mente que los desajustes de este tipo, aunque no sean percibidos de manera consciente, pueden influir negativamente en la imagen que el locutor nativo se forma de su interlocutor no nativo.

El consejo que cabe dar al angloparlante es usar, en el caso de la sugerencia concreta en cuestión, otras fórmulas convencionales, en particular una de las siguientes:

Hija, siéntate un rato a descansar.

¿Y si te sientas un rato a descansar?

Otra vez, al ser contrastadas explícitamente las preferencias de una y otra lengua se facilitaría el aprendizaje en mayor grado que dándole una "miniconferencia" al estudiante en la LM acerca de los distintos valores de cortesía que posee un conjunto de expresiones de esa lengua.

\section{G. Sociopragmática}

Se podría argumentar que en materias relativas a la pragmática de una LM, para lograr una comunicación eficiente, no es siempre cuestión de la simple elección de una expresión apropiada en la LM que exprese un determinado significado o matiz. Puede darse el caso de que en las socioculturas correspondientes a dos lenguas distintas existan divergentes preferencias sociopragmáticas. Podría tratarse, por ejemplo, de cuál nivel de cortesía se adecua a un determinado tipo de actividad. A título de ejemplo podría citarse una situación de compraventa en la que un hispanohablante nativo diría:

¿Me muestra la camisa azul del escaparate, por favor?

También le queda la opción de formularse ‘¿Podría hacerme el favor de mostrarme...?', aunque esto supondría una subida de nivel de cortesía, posiblemente interpretable como ironía (por ejemplo cuando el dependiente 
no le presta la debida atención al cliente). Lo que un angloparlante diría en la misma situación sería, por defecto, un giro que se pareciera a la fórmula española de mayor cortesía, o sea, algo como:

Could you please show me the blue shirt in the window?

Ahora bien, en ambos idiomas la pregunta hecha sin recurso al auxiliar 'can'/ poder ${ }^{4}$ resulta ser menos cortés que la variante que contiene el auxiliar en cuestión, lo cual nos hace sospechar que en por lo menos algunas socioculturas anglosajonas el nivel de cortesía usado convencionalmente en semejante tipo de situación sea más alto que en una mayoría de socioculturas hispánicas.

Contrastando ejemplos de este tipo se dilucidaría, no solo diferencias de expresiones verbales sino también conceptualizaciones divergentes de situaciones.

\section{A MODO DE CONCLUSIÓN}

La presente contribución se propone, por una parte, dar una visión de conjunto sobre un debate que desde hace varias décadas viene dedicado a los pros y los contras del uso de la LB en diversos tipos de enseñanza de segundas lenguas. Como parte de este debate entra la cuestión de la viabilidad o utilidad de un enfoque contrastivo aplicado a esta enseñanza. Creemos haber encontrado suficientes argumentos que apoyan el uso de la LB, no solo en la clase LE tradicional sino también en la clase de inmersión o de AICLE e incluso, bajo ciertas condiciones, en la enseñanza de LS del tipo "español para extranjeros", con estudiantes de LB mixtas.

Recurrir a la LB en la enseñanza de segundas lenguas resulta tener un potencial sumamente beneficioso para el aprendizaje en varios sentidos, en particular por abrir espacio a una perspectiva contrastiva que permite, no solo concientizar al estudiante sobre las divergencias y semejanzas entre la LB y la LM, sino también, y ante todo, simplificar las explicaciones dadas en el aula para así facilitar el aprendizaje de la LM. El enfoque contrastivo resulta ser de particular importancia en la enseñanza de lenguas de herencia, por tratarse de una "doble contrastividad" al emerger contrastes, no solo entre la lengua mayoritaria y la de herencia, sino también entre la LH y su correspondiente variedad estándar.

4 A la expresión 'me muestra...' le equivaldría en inglés, no una construcción con verbo en presente ('do you...') sino más bien con verbo en futuro 'will you show me....' 
Abstenerse de aplicar un enfoque contrastivo en la enseñanza de LE (o incluso en la enseñanza de LS) es privar al aprendiente del recurso probablemente más eficaz para alcanzar una competencia idiomática de la LM. Lo cual-claro-podría ser considerado un objetivo cuestionable por proponentes empedernidos de la exclusividad de la LM en el aula, quienes argumentarían que el objetivo principal de la enseñanza de LE/LS es que el aprendiente llegue nada más a hacerse entender. A lo cual se puede objetar que las ventajas sociales que el uso idiomático de la LM conlleva, representan un salto cualitativo decisivo en la comunicación con los usuarios nativos de la LM. Un argumento final y vital en pro de una utilización de la LB en el aula de segundas lenguas basada en un enfoque contrastivo es que el uso sistemáticamente paralelo de la LB y la LM ayuda al estudiante a adquirir y reforzar una identidad de individuo bi- o multilingüe. 
REFERENCIAS BIBLIOGRÁFICAS

Aronsson, Berit y Lars Fant (2014), Boundary tones in non-native speech: The transfer of pragmatic strategies from L1 Swedish into L2 Spanish. Intercultural Pragmatics 11(2), 159-198.

BARDovi-HarLIG, Kathleen y Zoltán Dörnyei (1998), Do Language Learners Recognize Pragmatic Violations? Pragmatic Versus Grammatical Awareness in Instructed L2 learning. Tesol Quarterly 32: 2, 233-259.

BeAUDRIE, Sara M. y Marta Fairclough (eds.) (2012), Spanish as a Heritage Language in the United States: The State of the Field. Georgetown: Georgetown University Press.

Behan, Laurie, Miles Turnbull, and J. Spek (1997), The proficiency gap in late French immersion: Language use in collaborative tasks. Le Journal de I'Immersion 20 (2), 41-44.

Brooks, Frank y Richard Donato (1994), Vygotskian approaches to understanding foreign language learner discourse during communicative tasks. Hispania 77, 262-274.

Byıund, Emanuel, Niclas Abrahamssony Kenneth Hyltenstam (2012), Does first language maintenance hamper nativelikeness in a second language? A study of ultimate attainment in early bilinguals. Studies in Second Language Acquisition 34, Special Issue 2, 215-241.

Canale, Michael y Merrill Swain (1980), Theoretical bases of communicative approaches to second language teaching and testing. Applied Linguistics 1, $1-47$.

Consejo de Europa/Council of Europe (2001), A Common European Reference Frameworkfor Languages: Learning, Teaching, Assessing [Marco Común Europeo de Referencias para el aprendizaje, enseñanza y evaluación de lenguas]. http://www.coe.int/t/dg4/education/elp/elpreg/Source/Key_reference/ CEFR_EN.pdf

Cummins, Jim (2000), Language, Power, and Pedagogy: Bilingual Children in the Crossfire. Clevedon: Multilingual Matters.

DAILEY-O'CAIN, Jennifer y Grit Liebscher (2009), Teacher and Student Use of the First Language in Foreign Language Classroom Interaction: Functions and Applications. En: M. Turnbull y J. Dailey-O'Cain (eds.), First Language Use in Second and Foreign Language Learning, 131-44. Bristol: Multilingual Matters. 
Diмrotн, Christine, Rebekah Rast, Marianne Starren y Marzena Watorek (2013), Methods for studying a new language under controlled input conditions: The VILLA project. Eurosla Yearbook 13, 109-138. Amsterdam: John Benjamins.

Dumitrescu, Domnita (2015), Teaching Spanish Heritage Speakers in the US: Challenges and rewards. PP-presentation at Stockholm University 18/09/2015.

Eluss, Nick (2005), At the interface: Dynamic interactions of explicit and implicit language knowledge. Studies in Second Language Acquisition 27, 305-352.

EluIS, Rod (2008), The Study of Second Language Acquisition. Oxford: Oxford University Press.

FaIRCLOUGH, Marta (2012), Language Assessment: Key Theoretical Considerations in the Academic Placement of Spanish Heritage Language Learners. En: S. M. Beaudrie y $\mathrm{M}$.

FAIRCLOUGH (eds.) (2012), Spanish as a Heritage Language in the United States: The State of the Field, 259-277. Georgetown: Georgetown University Press.

Fuller, Janet M. (2009), How Bilingual Children Talk: Strategic Codeswitching Among Children in Dual Language Programs. En: M.Turnbull y J. Dailey$\mathrm{O}$ 'Cain (eds.), First Language Use in Second and Foreign Language Learning, 115-30. Bristol: Multilingual Matters.

GarcíA, Ofelia (2008), Bilingual Education in the 21st Century. A Global Perspective. Hoboken NJ: Wiley-Blackwell.

Gaso, Laurent (2007), Linguistic Knowledge and Subject Knowledge: How Does Bilingualism Contribute to Subject Development? The International Journal of Bilingual Education and Bilingualism, 10: 5, 563-581.

(2015), Alternating languages and integrating language and content in bilingual education: theoretical and practical issues. PPpresentation at Stockholm University 01/10/2015.

GENESEE, Fred (1994), Integrating language and content: Lessons from immersion. Educational Practice Reports, 11. National Center for Research on Cultural Diversity and Second Language Learning, Washington, DC: Center for Applied Linguistics.

Krashen, Stephen D. (1981), Second Language Acquisition and Second Language Learning. New York: Pergamon.

(1982), Principles and Practice in Second Language Acquisition. New York: Pergamon.

(1985), The Input Hypothesis: Issues and Implications. London: 
LADO, Robert (1957), Linguistics across cultures: Applied linguistics for language teachers. Ann Arbor: University of Michigan Press.

Lugoloobi-Nalunga, Maureen (2013), Teaching and learning English in a multilingual classroom. A study of code-switching in an EFL/ESL teaching/learning situation. Degree Project. University of Karlstad, Department of Language, Literature and Intercultural Studies.

MACARO, Ernesto (2001), Analysing student teachers' codeswitching in foreign language classrooms: Theories and decision making. The Modern Language Journal 85 (4), 531-548.

(2009), Teacher Use of Codeswitching in the Second Language Classroom: Exploring 'Optimal' Use. En: M. Turnbull y J. Dailey-O'Cain (eds.), First Language Use in Second and Foreign Language Learning, 35-49. Bristol: Multilingual Matters.

MAJOR, Roy C. (2001), Foreign Accent: The Ontogeny and Phylogeny of Second Language Phonology. Mahwah: Lawrence Erlbaum.

McMillan, Brian y Miles Turnbull (2009),Teachers' Use of the First Language in French Immersion: Revisiting a Core Principle. En: M. Turnbull y J. Dailey$\mathrm{O}$ 'Cain (eds.), First Language Use in Second and Foreign Language Learning, 15-34. Bristol: Multilingual Matters.

NAGY, Krisztina y Daniel Robertson (2009). Target Language Use in English Classes in Hungarian Primary Schools. En: M. Turnbull y J. Dailey-O'Cain (eds.), First Language Use in Second and Foreign Language Learning, 66-86. Bristol: Multilingual Matters.

NoRRMAn, Gustav (2014), Förstaspråkets funktion i andraspråksundervisningen. En systematisk litteraturstudie för språkundervisning på vetenskaplig grund. Master's Degree Dissertation. Umeå University, Department of Applied Educational Studies.

Parodi, Carlos (2001, "El lenguaje de los proyectos". Gerencia social. Diseño, monitoreo y evaluación de proyectos sociales. Lima: Universidad del Pacífico.

SwalN, Merrill y Sharon Lapkin (2000) Task-based second language learning: The uses of the first language. Language Teaching Research 4, 253-276.

TronNIER, Mechtild y Elisabeth Zetterholm (2015), Patterns of prominence in L2: observations from learners of Swedish with L1s of diverse prominence properties.https://www.internationalphoneticassociation.org/icphsproceedings/ICPhS2015/ Papers/ICPHS0823.pdf 
TurnbulL, Miles y Jennifer Dailey-O'Cain (2009), Concluding Reflections: Moving Forward. En: M. Turnbull y J. Dailey-O'Cain (eds.), First Language Use in Second and Foreign Language Learning, 182-186. Bristol: Multilingual Matters.

TurnbulL, Miles y Jennifer Dailey-O'Cain (eds.) (2009), First Language Use in Second and Foreign Language Learning. Bristol: Multilingual Matters.

VALDÉs, Guadalupe (2012), Afterword: Future Directions for the Field of Spanish as a Heritage Language. En: S. M. Beaudrie y M. Fairclough (eds.), Spanish as a Heritage Language in the United States: The State of the Field, 279-289. Georgetown: Georgetown University Press.

VILLA, Daniel J. (2002), The Sanitizing of U.S. Spanish in Academia. Foreign Language Annals 35:2, 222-230.

VyGotsky, Lev S. (1978), Mind in Society: The Development of Higher Psychological Processes. Editado por M. Cole, V. John-Steiner, S. Scribner y E. Souberman Cambridge, MA: Harvard University Press. 
